\title{
PREVALENCIA DE ENFERMEDAD PERIODONTAL RELACIONADA CON PARTO PRETÉRMINO Y BAJO PESO AL NACER. HOSPITAL DE LA MUJER ALFREDO G. PAULSON 2017
}

\author{
PREVALENCE OF PERIODONTAL DISEASERELATED TO PRETERM LABOR AND LOW BIRTH \\ WEIGHT. HOSPITAL FOR WOMEN ALFREDO G. PAULSON 2017 \\ PREVALÊNCIA DE DOENÇA PERIODONTAL RELACIONADA AO TRABALHO PRÉ-MORTO E BAIXO PESO AO \\ NASCIMENTO. HOSPITAL PARA MULHERES ALFREDO G. PAULSON 2017
}

\author{
HURÍ DE ALBA CASTRO ARREAGA', GEOCONDA LUZARDO JURADO' \\ ${ }^{1}$ Universidad Católica de Santiago de Guayaquil; Guayaquil, Ecuador
}

RESUMEN

Introducción: la enfermedad periodontal (EP) es una infección multifactorial producida por bacterias aerobias y anaerobias que colonizan el surco gingival; los factores locales, genéticos, ambientales y biológicos favorecen la evolución del proceso destructivo, y en vía contraria, la EP afecta a las mujeres en estado de gestación debido a las citoquinas y otros mediadores inflamatorios que se diseminan por vía sanguínea hasta alcanzar la cavidad uterina, donde promoverán la síntesis de prostaglandinas (PGs), provocando estrés oxidativo y contracción del músculo liso, estos eventos están implicados en la historia natural del parto prematuro. Metodología: estudio de tipo transversal, observacional y descriptivo; se lo realizó en el hospital de la Mujer Alfredo G. Paulson de la ciudad de Guayaquil, con un universo de 930 pacientes. Se evaluaron 77 pacientes que cumplían con los criterios de inclusión, se les realizó un examen periodontal y se recopilaron datos del parto (semanas de gestación) y del neonato (peso al nacer). Resultados: en relación con la prevalencia de la enfermedad periodontal, el 62,34\% (48) presentaron salud periodontal, el 33,77\% (26) tenían gingivitis y solo el 3,90\% (3) padecían de periodontitis leve. Conclusiones: la enfermedad periodontal de las puérperas incluidas en el estudio no esta asociada al bajo peso al nacer.

PALABRAS CLAVE: enfermedad periodontal, parto pretérmino, bajo peso al nacer, prevalencia.

ABSTRACT

Introduction: periodontal disease (PD) is a multifactorial infection produced by aerobic and anaerobic bacteria that colonize the gingival sulcus. Local, genetic, environmental, and biological factors favor the evolution of the destructive process, and on the contrary, PD affects women in a state of pregnancy due to cytokines and other inflammatory mediators that spread through the blood until reaching the uterine cavity, where they will promote the synthesis of prostaglandins (PG), causing oxidative stress and smooth muscle contraction, these events are involved in the natural history of preterm birth. Methodology: cross-sectional, observational, and descriptive study was performed at the Alfredo G. Paulson Women's Hospital in the city of Guayaquil, with a universe of 930 patients. Seventy-seven patients who met the inclusion criteria were evaluated, underwent a periodontal examination, and data on delivery (gestation weeks) and neonate (birth weight) were collected. Results: concerning the prevalence of periodontal disease, $62.34 \%$ (48) presented periodontal health, 33.77\% (26) had gingivitis, and only 3.90\% (3) suffered from mild periodontitis. Conclusions: periodontal disease in the puerperal women included in the study is not associated with low birth weight.

KEYWORDS: periodontal disease, obstetric labor premature, low birth weight, prevalence.

RESUMO

Introdução: a doença periodontal (DP) é uma infecção multifatorial produzida por bactérias aeróbias e anaeróbias que colonizam o sulco gengival; Fatores locais, genéticos, ambientais e biológicos favorecem a evolução do processo destrutivo, ao contrário, a DP atinge mulheres em estado de gravidez devido a citocinas e outros mediadores inflamatórios que se espalham pelo sangue até atingir a cavidade uterina, onde irão. promover a síntese de prostaglandinas (PGs), causando estresse oxidativo e contração da musculatura lisa, esses eventos estão envolvidos na história natural do nascimento prematuro. Metodologia: estudo transversal, observacional e descritivo; Foi realizado no Hospital Feminino Alfredo G. Paulson, na cidade de Guayaquil, com um universo de 930 pacientes. Foram avaliadas setenta e sete pacientes que atenderam aos critérios de inclusão, submetidas a exame periodontal e coletados dados sobre o parto (semanas de gestação) e do recém-nascido (peso ao nascer). Resultados: em relação à prevalência de doença periodontal, 62,34\% (48) apresentavam saúde periodontal, 33,77\% (26) gengivite e apenas 3,90\% (3) periodontite leve. Conclusões: a doença periodontal nas puérperas incluídas no estudo não está associada ao baixo peso ao nascer.

PALAVRAS-CHAVE: doença periodontal, trabalho de parto prematuro, baixo peso de nascimento, prevalência. 
INTRODUCCIÓN

La enfermedad periodontal (EP) es una infección multifactorial producida por bacterias aerobias y anaerobias que colonizan el surco gingival. Diversos factores locales, genéticos, ambientales y biológicos favorecen la evolución del proceso destructivo, como el caso de las hormonas sexuales femeninas ya que su concentración aumenta considerablementeen el organismo a nivel vascular, celular, microbiológico einmunitario. ${ }^{1-3}$ En una primera vía, las citoquinas $\mathrm{y}$ otros mediadores inflamatorios generados en la respuesta inmune frente a la infección se diseminarían por vía sanguínea hasta alcanzar la cavidad uterina, donde promoverán la síntesis de prostaglandinas (PGs). Entre los diversos efectos producidos por estos mediadores de la inflamación se encuentran los siguientes: la prostaglandina E2 (PGE2) provoca estrés oxidativo, contracción del músculo liso y oxidación de las lipoproteínas de baja densidad (LDL), mientras quela interleuquina $1 \beta$ (IL-1 $\beta$ ), el factor de necrosis tumoral $\alpha$ (TNF $\alpha)$ y la interleuquina 6 (IL-6) son capaces de estimular la adhesión endotelial, hiperlipidemia, liberación hepática de reactivos de fase aguda y catabolismo del tejido conectivo entre otros efectos. Muchos de estos eventos están implicados en la historia natural del parto prematuro (Figura 1), que según la Organización Mundial de la Salud define como prematuro a aquel nacimiento de menos de 37 semanas, muy prematuro menos de 32 semanas y como bajo peso al nacer menor a $2500 \mathrm{~g}$., muy bajo peso al nacer menos de $1500 \mathrm{~g}$. y extremadamente bajo peso menos de $1000 \mathrm{~g}$. $^{4-6}$

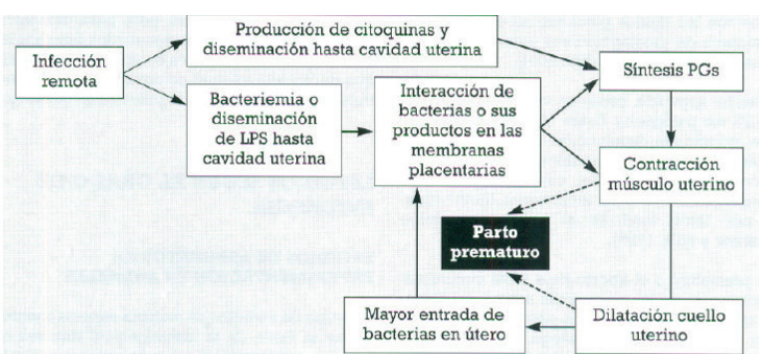

Figura 1. Mecanismo mediante el cual una infección remota puede afectar el embarazo y desencadenar el parto prematuro.

Con la presente investigación se propone reconocer la prevalencia de la enfermedad periodontal en mujeres puérperas con relación al bajo peso al nacer y parto pretérmino y estimular medidas de prevención.

\section{METODOLOGÍA}

El presente estudio es de tipo transversal, observacional y descriptivo; se lo realizó en el hospital de la Mujer Alfredo G. Paulson de la ciudad de Guayaquil, con un universo de 930 pacientes.
Dentro de los criterios deinclusión se seleccionaron:

1. Mujeres puérperas que tuvieron un parto pretérmino (<37 semanas de gestación) y/o que dieron a luz un niño con bajo peso $(<2500 \mathrm{~g})$

2. Mujeres puérperas de $14-29$ años y 30-45 años.

Se excluyeron del estudio:

1. Mujeres puérperas con un tiempo de embarazo adecuado (38-40 semanas de gestación).

2. Mujeres puérperas menores de 14 años y mayores de 45 años.

Para el presente estudio se evaluaron 77 pacientes que cumplían con los criterios de inclusión y se procedió a firmar el consentimiento informado y a llenar la hoja de registro en donde se recopilaron datos personales del parto y del neonato, para la recolección de estos datos fueron usadas las historias clínicas de cada paciente.

Luego de la toma de datos se realizó un examen clínico periodontal en las 48 horas posteriores al parto, donde se utilizó un kit de diagnóstico básico y una sonda periodontal Carolina del Norte. Se efectuará el análisis de datos con el paquete estadístico SPSS. Se harán las comparaciones pertinentes con las pruebas según la normalidad de la distribución de los datos. El objetivo de este estudio es determinar la prevalencia de la enfermedad periodontal relacionado al parto pretérmino y bajo peso al nacer.

\section{RESULTADOS}

Luego de tomar en cuenta los criterios de inclusión y excusión se evaluó una muestra de 77 mujeres puérperas, en los siguientes rangos de edad: el $58,44 \%$ (45) estaban dentro de un rango de edad de 14 a 29 años, mientras que el 41,56\% (32) eran entre 30 a 45 años.

En relación con la prevalencia de la enfermedad periodontal en la tabla 1 , se puede observar que el 62,34\% (48) presentaron salud periodontal, el $33,77 \%$ (26) tenían gingivitis y solo el 3,90\% (3) padecían de periodontitis leve.

TABAL 1. CUADRO DE PREVALENCIA DE ENFERMEDAD PERIODONTAL ENFERMEDAD PERIODONTAL

\begin{tabular}{lrr} 
Salud & 48 & 62,34 \\
\hline Gingivitis & 26 & 33,77 \\
\hline Periodontitis leve & 3 & 3,90 \\
\hline Periodontitis moderada & 0 & 0,00 \\
\hline Periodontitis severa & 0 & 0,00 \\
\hline Total & 77 & 100
\end{tabular}


En la frecuencia del peso al nacer de los neonatos, el $42,86 \%$ (33) presentaron bajo peso, 27,27\% (21) muy bajo peso yel 24,68\%(19) peso extremadamente bajo. En cuanto al tipo de parto, el 51,95\% (40) fueron partos muy prematuros, $38,96 \%$ (30) prematuros, mientras que solo el 9,09\% (7) tuvo un desenlace a término.

Al asociar el estado periodontal y peso del niño al nacer (tabla), se encontró que del 100\% (48) de las puérperas que presentaban salud periodontal; el 47,92\% (23) de los neonatos tenían un peso bajo, $27,08 \%$ (13) peso muy bajo, 20,83\% (10) peso extremadamente bajo y solo el $4,17 \%$ (2) un peso normal. Por otrolado, del $100 \%$ (26) de las puérperas que presentaron gingivitis; el 38,46\% (10) de los neonatos presentó un peso bajo, 30,77\% (8) peso extremadamente bajo, 23,08\% (6) peso muy bajo y el 7,69\% (2) un peso normal.

TABLA 2. CUADRO DE CORRELACIÓN ENTRE ESTADO PERIODONTAL Y PESO DEL NIÑO AL NACER

\begin{tabular}{lrrrrrr} 
& \multicolumn{2}{c}{ SALUD } & \multicolumn{2}{c}{ GINGiVITIS } & \multicolumn{2}{c}{ PERIODONTITIS LEVE } \\
Normal & $4,17 \%$ & 2 & $7,69 \%$ & 2 & $0,00 \%$ & 0 \\
\hline Bajo peso & $47,92 \%$ & 23 & $38,46 \%$ & 10 & $0,00 \%$ & 0 \\
\hline Muy bajo peso & $27,08 \%$ & 13 & $23,08 \%$ & 6 & $66,67 \%$ & 2 \\
\hline $\begin{array}{l}\text { Extremadamente } \\
\text { bajo peso }\end{array}$ & $20,83 \%$ & 10 & $30,77 \%$ & 8 & $33,33 \%$ & 1 \\
\hline Total & $100,00 \%$ & 48 & $100,00 \%$ & 26 & $100,00 \%$ & 3
\end{tabular}

Mientras que, del 100\% (3) de las puérperas que presentaron periodontitis leve; el 66,67\% (2) de los neonatos fueron de peso muy bajo y el 33,33\% (1) extremadamente bajo. Cabe recalcar que no se presentó periodontitis moderada y severa.

En la tabla 3, relacionando el estado periodontal con el tipo de parto, los autores señalan que del $100 \%$ (48) de las puérperas que presentaban salud periodontal; el 47,92\% (23) tuvieron partos muy prematuros y un $39,58 \%$ (19) fueron prematuros. A su vez, del $100 \%$ (26) de las mujeres puérperas que presentaron gingivitis, un 53,85\% (14) partos muy prematuros y el $42,31 \%$ (11) eran partos prematuros. Del $100 \%$ (3) que presentaron periodontitis leve; el $100 \%$ (3) tuvo un desenlace muy prematuro.

TABLA 3. CUADRO DE CORRELACIÓN ENTRE ESTADO PERIODONTAL Y TIPO DE PARTO

\begin{tabular}{lrrrrrr} 
& \multicolumn{1}{c}{ SALUD } & & GINGIVITIS & \multicolumn{2}{c}{ PERIODONTITIS LEVE } \\
\hline A término & $12,50 \%$ & 6 & $3,85 \%$ & 1 & $0,00 \%$ & \\
\hline Prematura & $39,58 \%$ & 19 & $42,31 \%$ & 11 & $0,00 \%$ & \\
\hline Muy prematuro & $47,92 \%$ & 23 & $53,85 \%$ & 14 & $100,00 \%$ & 3 \\
\hline Totales & $100,00 \%$ & 48 & $10,00 \%$ & 26 & $100,00 \%$ & 3
\end{tabular}

En la tabla 4, el estado periodontal versus la edad de las puérperas se encontró que del $100 \%$ (48) de las mujeres puérperas que presentaban salud periodontal, el 62,50\% (30) eran de edades entre 14 a 29 años y 37,50\%(18) de 30 a 45 años. Sin embargo, del $100 \%$ (26) de las puérperas que presentaron gingivitis, el 53,85\% (14) eran de edades entre 30 a 45 años y un $46,15 \%$ (12) de 14 a 29 años. Por otra parte, del 100\% (3) de las mujeres que presentaron periodontitis leve; el 100\%(3) era de 14 a 29 años.

TABLA 4. CUADRO DE CORRELACIÓN ENTRE ESTADO PERIODONTAL CON EDAD MATERNA

\begin{tabular}{lrrrrrr} 
& \multicolumn{1}{c}{ SALUD } & \multicolumn{2}{c}{ GINGIVITIS } & \multicolumn{2}{c}{ PERIODONTITIS LEVE } \\
\hline $14-29$ años & $62,50 \%$ & 30 & $46,15 \%$ & 12 & $100,00 \%$ & 3 \\
\hline $30-45$ años & $37,50 \%$ & 18 & $53,85 \%$ & 14 & $0,00 \%$ & 0 \\
\hline Totales & $100,00 \%$ & 48 & $100,00 \%$ & 26 & $100,00 \%$ & 3
\end{tabular}

En la tabla 5, de la relación del peso del niño al nacer con la edad de la madre, se encontró que del $100 \%$ (33) de los neonatos con bajo peso; el 60,61\% (20) eran mujeres puérperas entre 14 a 29 años y un 39,39\% (13) entre 30 a 45 años. Así mismo, del $100 \%$ (21) de los neonatos con un peso muy bajo; el $61,90 \%$ (13) eran mujeres puérperas de edades entre 14 a 29 años, y un 38,10\% (8) entre 30 a 45 años. Por otra parte, del 100\%(19) de los neonatos con extremadamente bajo peso; el 52,63\% (10) eran mujeres puérperas de 30 a 45 años, y 47,37\% (9) entre 14 a 29 años.

\section{TABLA 5. CUADRO DE CORRELACIÓN ENTRE PESO DEL NEONATO Y EDAD} MATERNA

\begin{tabular}{lrrrrrr} 
& $\begin{array}{r}\text { BAJO PESO } \\
\mathbf{2} \mathbf{2 5 0 0 g}\end{array}$ & $\begin{array}{r}\text { BAJO PESO }< \\
\mathbf{1 5 0 0 g}\end{array}$ & $\begin{array}{r}\text { EXTREMADAMENTE } \\
\text { BAJO PESO }< \\
\mathbf{1 0 0 0 g}\end{array}$ \\
\hline $14-29$ años & $60,61 \%$ & 20 & $61,90 \%$ & 13 & $47,37 \%$ & 9 \\
\hline $30-45$ años & $69,39 \%$ & 13 & $38,10 \%$ & 8 & $52,63 \%$ & 10 \\
\hline Totales & $100,00 \%$ & 33 & $100,00 \%$ & 21 & $100,00 \%$ & 19
\end{tabular}

Encuantoal tipo departo con la edad delas mujeres, se pudo evidenciar que del $100 \%$ (30) de las mujeres que tuvieron un parto prematuro; el 56,67\% (17) eran mujeres entre los 14 a 29 años, y 43,33\% (13) entre los 30 a 45 años. De la misma forma, del $100 \%$ (40) de mujeres puérperas que tuvieron un parto muy prematuro; $52,50 \%$ (21) fueron mujeres entre los 14 a 29 años, mientras que 47,50\% (19) de 30 a 45 años.

DISCUSIÓN

En 1996, Offenbacher y su grupo de la Universidad de Carolina del Norte evidenciaron la asociación entre periodontitis y parto prematuro (PP) / bajo 
peso al nacimiento (BPN) determinando 124 mujeres embarazadas o postparto; las mujeres con PP/BPN mostraron una pérdida de inserción significativamentemayor, situandoa la enfermedad periodontal como factor de riesgo para $\mathrm{PP} / \mathrm{BPN}$ con un riesgo relativo (RR) de 7.9. ${ }^{4}$ En 2004 Coepfterd et al., llevaron a cabo un estudio caso control con 59 mujeres que habían sufrido de parto prematuro antes de las 32 semanas de gestación, en una población control de 44 mujeres con un parto normal. Examinaron el nivel de enfermedad periodontal y encontraron que las gestantes que presentan enfermedad periodontal severa son 3 veces más propensas a sufrir un parto prematuro espontáneo. ${ }^{7}$ En un estudio realizado por Castaldi et al., en 2006 reveló que el 51,8\% de las mujeres presentaban alguna afección bucal; de ellas, el 17,5\% padecían EP grave y el 34,3\%, gingivitis.

Eldesenlace de los partos; 9,5\% fueron pretérmino, el $10,3 \%$ nacieron niños con bajo peso y el $10,0 \%$ la paciente sufrió preeclampsia. ${ }^{3}$ En otro estudio observacional, transversal y analítico realizado por García G., Vega S. y Tolentino A., en 2016 se consideró que la prevalencia de enfermedad periodontal y placa bacteriana en las mujeres puérperas es de un $65 \%$ (16), demostrando que la prevalencia de enfermedad periodontal en embarazadas es un problema de salud pública a nivel mundial. ${ }^{8}$

Roca A y Pareja V. M. en el 2008, examinó 100 puérperas, 16 presentaron enfermedad periodontal, de las cuales sólo una tuvo un niño con bajo peso al nacer.

De las 84 madres sin enfermedad periodontal, 10 tuvieron niños con bajo peso al nacer. Al comparar con los resultados de este estudio encontramos que delas puérperasque presentaban salud periodontal; el $47,92 \%$ de los neonatos tenían un peso bajo. Por otro lado, de las puérperas que presentaron gingivitis; el 38,46\% (10) de los neonatos presentó un peso bajo. Mientras que, de las puérperas que presentaron periodontitis leve; el 66,67\% (2) de los neonatos fueron de peso muy bajo. Cabe recalcar que no se presentó periodontitis moderada y severa. ${ }^{9}$ Así mismo, en el estudio de Zermeño Jy cols. 2011 en un total de 282 pacientes, $48(15,25 \%)$ mujeres fueron diagnosticadas con enfermedad periodontal y $234(84,75 \%)$, se consideraron sanas. Asociando las complicaciones perinatales con la enfermedad periodontal se observó que $10 \%$ de las pacientes sanas presentaron parto pretérmino; mientras que en el grupo de pacientes enfermas presentaron 6,9\% parto pretérmino. Al comparar con los resultados de este estudio se afirma que el $47,92 \%$ de las mujeres con salud fueron prematuros. A su vez, $42,31 \%$ de las pacientes con gingivitis fueron partos prematuros. ${ }^{10}$

Contrastando esteúltimo, en el estudio de Gandri C y cols 2010, se seleccionó un total de 53 casos y 79 controles para este estudio. La mayoría de las madres pertenecía a la franja etaria de 20 a 34 años $(65,1 \%)$, aunque en promedio, los casos exhibieran una edad mayor que los controles. Las madres del grupo decasos $>35$ años presentaron una proporción mayor de factores de riesgo de parto pretermino o bajo peso al nacer $(20 \%)$, a diferencia en el presente estudio, en el que el $56.67 \%$ del grupo de mujeres puérperas entre 16 a 29 años presentaron parto pretérmino. ${ }^{1}$

\section{CONCLUSIÓN}

Seevidencióqueno existeuna diferencia significativa entreel estado periodontal delas mujeres puérperas que fueron incluidas en el estudio en relación con su extensión y severidad con el parto pretérmino y bajo peso al nacer, probablemente porque en el hospital de la mujer Alfredo G. Paulson, las pacientes en estado de gestación tienen un control prenatal odontológico previo. También se cree que las mujeres mayores a los 35 años tienen más probabilidades de tener hijos con un peso bajo al nacer o sufrir un parto pretérmino.

Sin embargo, para las siguientes investigaciones este tema debe ser evaluado cautelosamente ya que hay estudios que afirman que la EP si es un factor de riesgo para estas complicaciones. Se recomienda aumentar el número de muestra y tomar en cuenta dentro de los criterios de exclusión otros factores que puedan influenciar el parto pretérmino y bajo peso al nacer de los neonatos.

\section{REFERENCIAS BIBLIOGRÁFICAS}

1. Govindaraju P, Venugopals, Shivakumar MA, Sethuraman S, Ramaiah SK, Mukundan S. Maternal periodontal disease and preterm birth: A case-control study. J Indian Soc Periodontol. 2015;19(5):512-5.

2. Herane B, Godoy C, Herane C. Enfermedad periodontal y embarazo. Revisión de la literatura. Rev Médica Clínica Las Condes [Internet]. 2014;25(6):936-43. Available from: http://www.sciencedirect.com/science/article/ pii/S0716864014706418

3. Castaldi JL, Bertin MS, Giménez F, Roberto L. Enfermedad periodontal: ies factor de riesgo 
para parto pretérmino, bajo peso al nacer o preeclampsia? Rev panam salud pública [Internet]. 2006;19(1):253-8. Available from: http://www.scielosp.org/scielo.php?script=sci_ar ttext\&pid=S1020-49892006000400005

4. Flores J, Oteo A, Mateos L, Bascones A. Relación entreenfermedad periodontaly partoprematuro. Bajo peso al nacimiento: una revisión de la literatura. Av en Periodoncia e Implantol Oral [Internet]. 2004;16(2):93-105. Available from: http://scielo.isciii.es/scielo.php?script=sci arttext\&pid=S1699-65852004000200004\&ln $\mathrm{g}=\mathrm{es} \& \mathrm{nrm}=\mathrm{iso} \& \mathrm{tlng}=\mathrm{es}$

5. Meza Casso I, Garza Salinas B, Martínez Sandoval G, Rodríguez Franco N. Relación de la enfermedad periodontal y tratamiento periodontal con el parto pretérmino. Rev Mex Periodontlogía [Internet]. 2013;4(2):78-86. Available from: http://www.medigraphic.com/ pdfs/periodontologia/mp-2013/mp132f.pdf

6. Gutiérrez Romero F, Pareja Vásquez M. Parto Prematuro Y Bajo Peso Al Nacer Y Su Relación Con La Enfermedad Periodontal. Rev Kiru [Internet]. 2010;7(1):43-9. Available from: http://www.usmp.edu.pe/odonto/servicio/2010/ Kiru2010v7n1/kiru2010v7n1art8.pdf

7. Rivas A, Rivero C, Salas M, Urbina G, Villanueva J. Enfermedades periodontales en mujeres embarazadas : Artículo de revisión. Rev Venez Invest Odont IADR. 2014;2(1):60-75.

8. García-Morales G, Vega-Vega SP, TolentinoAlvarado A. Prevalencia de enfermedad periodontal en embarazadas de una unidad de medicina familiar de Acapulco, Guerrero. Atención Fam [Internet]. 2016;23(3):75-9. Available from: http://linkinghub.elsevier. com/retrieve/pii/S1405887116301286

9. Malttos AR, Vásquez MP. Enfermedad periodontal como factor de riesgo de bajo peso al nacer. Rev Kiru. 2008;5(2):111-5.

10. Zermeño N. J de J, Flores A. C del C, Saldívar R. D, Soria L. JA, Garza R. M, Iglesias B. JL. Enfermedad periodontal como factor de riesgo para presentar resultados perinatales adversos. Rev Chil Obs Ginecol [Internet]. 2011;76(5):338-43. Available from: http:// www.scielo.cl/scielo.php?script=sci_arttext \&pid=S0717-75262011000500009

11. Gazolla CM, Ribeiro A, Moysés MR, Oliveira LAM, Pereira LJ, Sallum AW. Evaluation of the Incidence of Preterm Low Birth Weight in Patients Undergoing Periodontal Therapy. J Periodontol [Internet]. 2007;78(5):842-8. Available from: http://www.joponline.org/ doi/10.1902/jop.2007.060295
12. Duque A, Tirado M, Arbeláez C, García S. Conocimientos y actitudes sobre la posible asociación de la periodontitis como factor de riesgo de algunas enfermedades y condiciones médicas en una muestra de médicos en Medellín-Colombia. (Knowledgeand Attitudes about the possible association of...). CES Odontol [Internet]. 2011;24(1):29-36. Available from: http://revistas.ces.edu.co/index.php/ odontologia/article/view/1472

13. Heras Pérez B, Gobernado Tejedor J, Mora Cepeda P, Almaraz Gómez A. La edad materna como factor de riesgo obstétrico. Resultados perinatales en gestantes de edad avanzada. Progresos Obstety Ginecol. 2011;54(11):575-80.

14. Comes-Filho IS, Cruz SS, Rezende EJC, Dos Santos CAST, Soledade KR, Magalhães $\mathrm{MA}$, et al. Exposure measurement in the association between periodontal disease and prematurity/low birth weight. J Clin Periodontol. 2007;34(11):957-63.

15. G-FIS, - da CSS, - REJ, - da SBB, - TSC, - PJS, et al. - Periodontal status as predictor of prematurity and low birth weight.; - Journal of public health dentistry. - J Public Heal Dent. 2006;66(4):295-8.

16. Khader YS, Ta’ani Q. Periodontal Diseases and the Risk of Preterm Birth and Low Birth Weight: A Meta-Analysis. J Periodontol. 2005;76(February):161-5.

17. Cohen L, Schaeffer M, Davideau J, Tenenbaum $\mathrm{H}$, Huck O. Obstetric knowledge, attitude, and behavior concerning periodontal diseases and treatment needs in pregnancy: influencing factors in France. J Periodontol [Internet]. 2015;86:398-405. Available from: http://ovidsp. ovid.com/ovidweb.cgi? T=JS\&CSC=Y\&NEWS $=\mathrm{N} \& \mathrm{PACE}=$ fulltext $\& D=$ prem $\& A N=25427617$ http://oxfordsfx.hosted.exlibrisgroup.com/ oxford?sid=OVID:medline\&id=pmid:254276 17\&id=doi:10.1902\%2Fjop. $2014.140371 \&$ issn $=0022-3492 \&$ isbn $=\&$ volume $=86 \&$ issue $=3 \& \mathrm{~s}$ page $=398$ \&page

18. Bassani DG, Olinto MTA, Kreiger N. Periodontal disease and perinatal outcomes: A case-control study. J Clin Periodontol. 2007;34(1):31-9.

19. Kim AJ, Lo AJ, Pullin DA, Thornton-Johnson DS, Karimbux NY. Scaling and root planing treatment for periodontitis to reduce preterm birth and low birth weight: a systematic review and meta-analysis of randomized controlled trials. J Periodontol [Internet]. 2012;83(12):1508-19. Available from: http:// www.ncbi.nlm.nih.gov/pubmed/22376207 
20. Dasanayake a P. Poor periodontal health of the pregnant woman as a risk factor for low birth weight. Ann Periodontol [Internet]. 1998;3(1):206-12. Available from: http://www. ncbi.nlm.nih.gov/pubmed/9722704

21. Heimonen A, Janket S-J, Kaaja R, Ackerson LK, Muthukrishnan P, Meurman JH. Oral Inflammatory Burden and Preterm Birth. J Periodontol [Internet]. 2009;80(6):884-91. Available from: http://www.joponline.org/ doi/10.1902/jop.2009.080560
22. Offenbacher S, KatzV, Fertik G, Collins J, Boyd D, Maynor G, et al. Periodontal infection as a possiblerisk factor for preterm low birth weight. J Periodontol. 1996;67(10 Suppl):1103-13. 